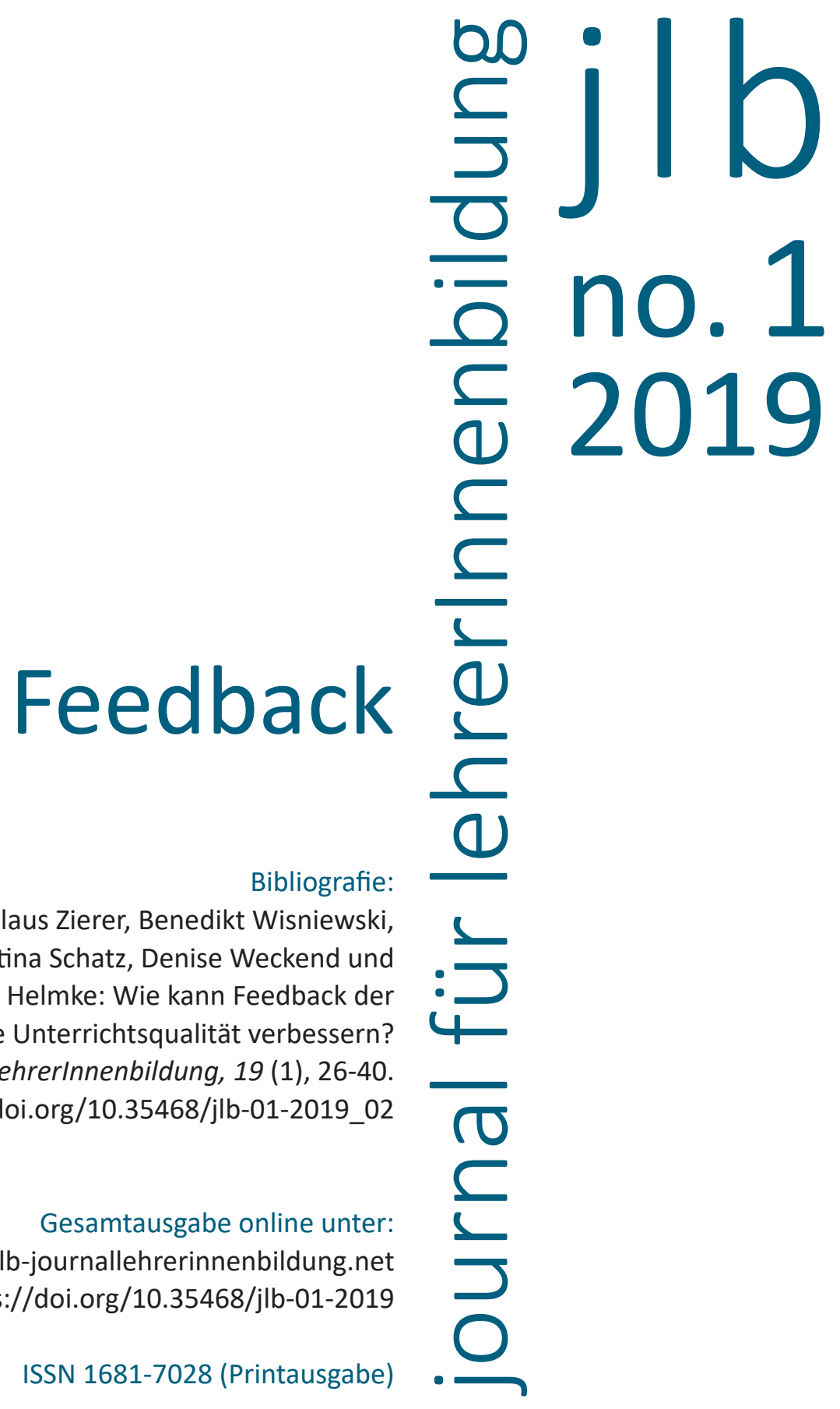


02

Klaus Zierer, Benedikt Wisniewski, Christina Schatz, Denise Weckend und Andreas Helmke

\section{Wie kann Feedback der Lernenden die Unterrichtsqualität verbessern?}


„Mehr als neuntausend Würfe in meiner Karriere gingen daneben. Ich habe fast dreihundert Spiele verloren. Sechsundzwanzig Mal lag es an mir, den spielentscheidenden Wurf zu machen, und ich habe versagt. Immer und immer und immer wieder bin ich in meinem Leben gescheitert. Und deswegen habe ich Erfolg." Mit diesen Worten fasst einer der weltbesten Basketballspieler aller Zeiten, Michael Jordan, seine professionelle Haltung zusammen. Er fordert dazu auf, bereit zu sein, aus Fehlern zu lernen und jeden Tag daran zu arbeiten, besser zu werden. Im schulischen Kontext tauchen häufig eher negativ konnotierte Begriffe (z. B. „Optimierungswahn“, nach Zellner, 2013) auf, wenn es darum geht, wie Lehrer*innen ${ }^{1}$ besser werden können in dem, was sie täglich tun. Dadurch, dass die Ergebnisse von Lehrprozessen nicht nur vom Angebot abhängen, das Lehrpersonen machen, sondern auch von der Nutzung dieses Angebots durch die Schüler*innen (Helmke, 2017), sind unmittelbare und eindeutige Rückmeldungen über die Ergebnisse der eigenen Arbeit - wie sie Michael Jordan zur Verfügung stehen - Lehrpersonen in dieser Form nicht zugänglich. Der Lernerfolg von Schüler*innen ist kein wöchentlich messbares Ergebnis, das sich monokausal erklären lässt. Allerdings bedeutet dies nicht, dass es Lehrer*innen an Rückmeldungen zur Qualität ihrer Arbeit mangelt, denn Rückmeldungen erhalten Lehrer*innen tagtäglich - häufig werden sie bloß nicht als solche wahrgenommen. Umso wichtiger erscheint es, eine Grundlage zu schaffen, um das professionelle Lehrpersonenhandeln auf der Basis von Daten reflektieren zu können. In diesem Beitrag wird mit Hilfe von Ergebnissen der empirischen Bildungsforschung zunächst ein Blick auf die Wirksamkeit von Feedback für schulische Lernprozesse geworfen. Darauf aufbauend wird in einem zweiten Schritt gezeigt, wie erfolgreiches Feedback mit Lehrer*innenprofessionalität in Verbindung steht, um in einem letzten Schritt Handlungsmöglichkeiten für die Schul- und Unterrichtspraxis anzuführen.

1 Im Folgenden verstehen wir unter Lehrer*innen Lehrende im Primar-, Sekundärund Tertiärbereich 


\section{Zur Wirksamkeit von Feedback}

Die Annäherung an den Forschungsstand zum Thema „Feedback" ist angesichts der Fülle an Studien herausfordernd. Wirft man beispielsweise einen Blick in John Hatties international bekanntes Werk „Visible Learning" und auf die aktuell 255 Faktoren (vgl. Hattie \& Zierer, 2018a), so wird deutlich: Feedback zählt nicht nur zu den am besten erforschten Methoden (31 Meta-Analysen aus 1.431 Einzelstudien über einen Zeitraum von 35 Jahren), sondern hat mit einer Effektstärke von $d=0,70$ zudem große Einflüsse auf die Lernleistung von Schüler*innen (vgl. Abb. 1).

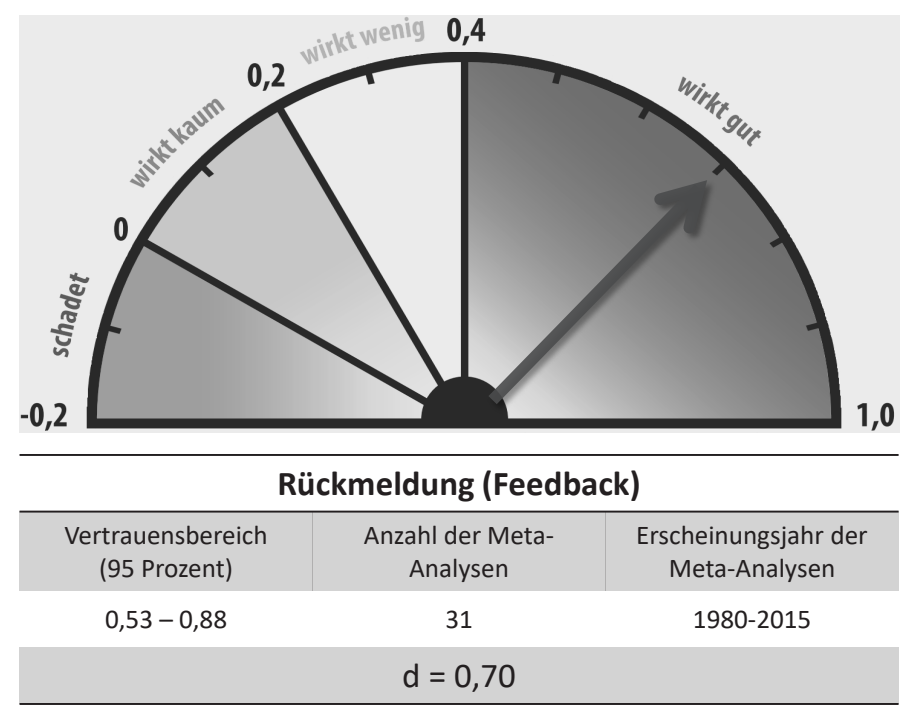

Abb. 1 Der Faktor „Rückmeldung (Feedback)“ in „Visible Learning“

Trotz dieser vielfältigen Ergebnisse lassen sich mindestens folgende grundlegende Aussagen zur Wirkung von Feedback formulieren:

(1) Erfolgreiches Feedback berücksichtigt verschiedene Ebenen, wobei im Hinblick auf Lernleistungen vor allem die Ebene der Aufgabe, des Prozesses und allen voran die Ebene der Selbstregulation entscheidend sind (vgl. Abb. 2 nach Hattie \& Zierer, 2018b). 

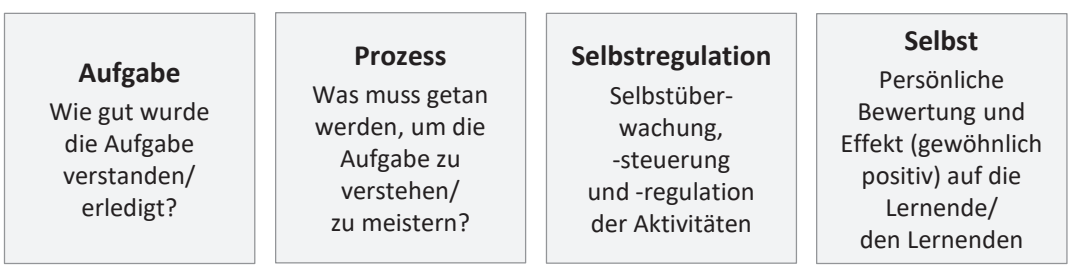

Abb. 2 Ebenen des Feedbacks

(2) Erfolgreiches Feedback versteht sich immer als Dialog, der sich nicht nur von der Lehrperson an die Lernenden richtet, sondern auch umgekehrt (vgl. Abb. 3, Hattie \& Zierer, 2018b). Dabei stellen das Feedbackgeben und das Feedbacknehmen die zwei entscheidenden Kompetenzen im Feedbackprozess dar, wobei sowohl die Lehrperson wie auch die Lernenden selbst die Rolle der Feedbackgebenden übernehmen können.

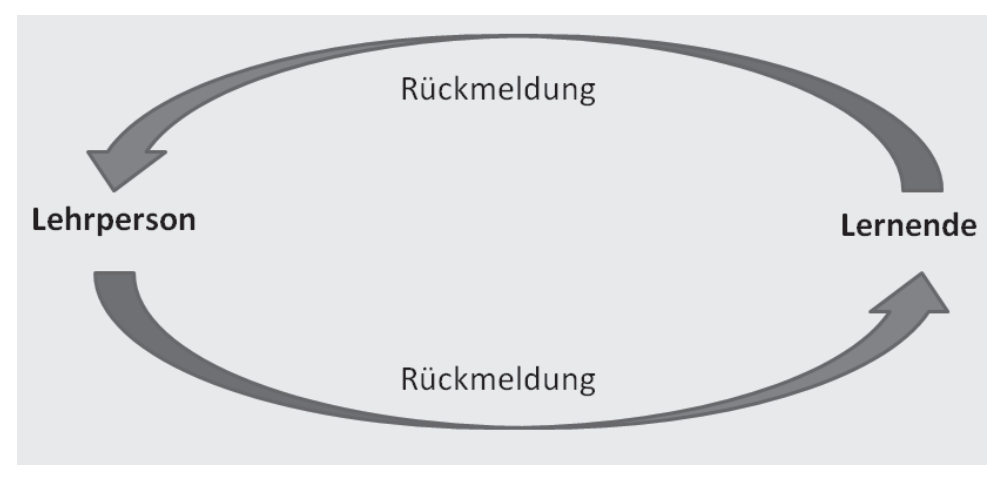

Abb. 3 Feedback als dialogischer Prozess

Gerade wenn Lernende der Lehrperson Feedback geben, kann das wirksam für erfolgreiches, herausforderndes und wertschätzendes Unterrichten sein. Die Lehrperson erfährt so, auf welchem Leistungsstand die Lernenden stehen, wo sie Verständnisprobleme haben und welche Fehler gemacht werden. Dadurch „können Lehren und Lernen miteinander synchronisiert werden" und die Diskrepanz zwischen Fremd- und Selbsteinschätzung von Unterricht wird reduziert (Hattie, 2018, S. 206). Rückmeldungen zur Wirksamkeit ihres Unterrichts können Lehrpersonen somit helfen, ihre didaktischen Entscheidungen 
zu überdenken, so dass Feedback ein Instrument zur Entwicklung von Unterricht wird. Das Feedback von den Lernenden zur Lehrperson stellt eine zentrale Komponente gelingenden Unterrichts dar, wie auch John Hattie (ebd.) unterstreicht: „Der Fehler, den ich machte, war, in Feedback etwas zu sehen, was die Lehrpersonen den Lernenden geben [...]. Erst als ich entdeckt habe, dass Feedback besonders wirksam ist, wenn es der Lehrperson von den Lernenden gegeben wird, begann ich, es besser zu verstehen." John Hatties Aussage lässt sich anhand der Einzelstudien, die in "Visible Learning“ eingeflossen sind, empirisch nachweisen - aufgrund mangelnder Datenlage aber nur an den Einzelstudien aus dem Tertiärbereich, die insgesamt eine durchschnittliche Effektstärke von 0,52 aufweisen und somit etwas niedriger sind als der Gesamteffekt in „Visible Learning“ von 0,70: Ein Vergleich mit dem durchschnittlichen Effekt aus 392 Primärstudien zum Feedback, das Lehrpersonen Lernenden geben, zeigt, dass beide Formen ähnlich effektiv sind, wenngleich die Streuung der Primärstudieneffekte beim Lernenden-Lehrenden-Feedback deutlich höher ausfällt (vgl. Abb. 4). Dies ist an den Konfidenzintervallen abzulesen. Die hohe Streuung weist darauf hin, dass die Wirksamkeit für diese Form des Feedbacks offensichtlich stark durch Merkmale der Umsetzung moderiert wird.

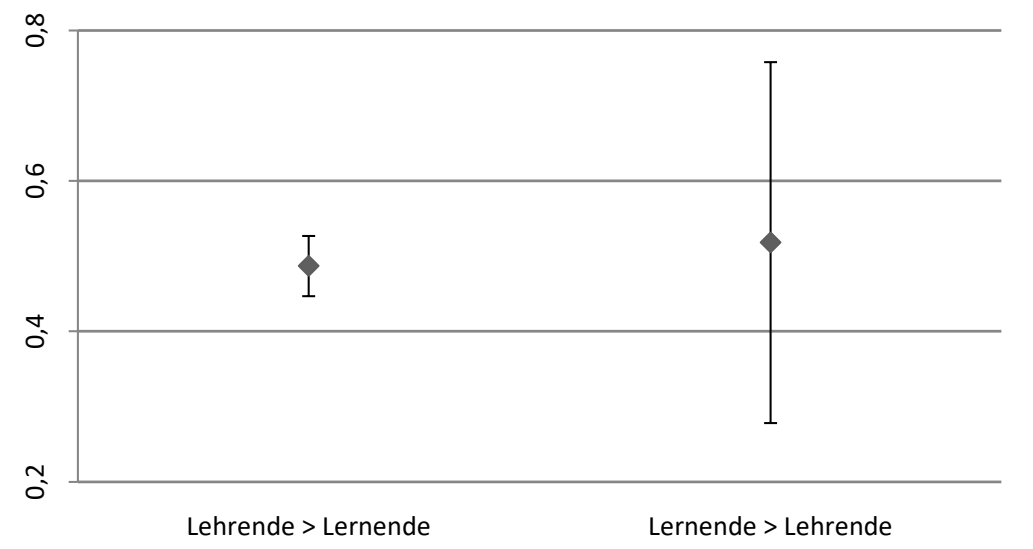

Richtung des Feedbacks

Abb. 4 Durchschnittliche Effektstärken von Feedback zwischen Lehrenden und Lernenden (Wisniewski \& Zierer, 2019) 
(3) Die Wirkung von Feedback an Lehrpersonen auf die Unterrichtsqualität ist empirisch gut belegt (Ditton \& Arnold, 2004; Gärtner, 2013; Bastian, Combe \& Langer, 2004; Buhren \& Reitz, 2007; Helmke, 2017) und stellt eine wesentliche Bedingung für zielführende Selbstreflexionsprozesse dar. Entscheidend für gelingende Feedbackprozesse ist die Bereitschaft, das erhaltene Feedback auch anzunehmen und für die eigene Entwicklung nutzbar zu machen. Erfolgreiches Feedback ist also nicht nur eine Frage der Kompetenz, sondern in entscheidender Weise auch abhängig von der Haltung gegenüber Feedback. Dies wird deutlich, wenn man beispielsweise die DESI-Video-Studie (Helmke, Helmke, Schrader, Wagner, Nold \& Schröder, 2008) näher betrachtet: Lehrpersonen schätzten ihren Sprechanteil in einer Unterrichtsstunde selbst ein, und gleichzeitig wurde dieser auf der Basis von Video-Aufnahmen objektiv gemessen. Die Selbsteinschätzungen lagen deutlich unter den tatsächlich gemessenen Anteilen (vgl. Abb. 5). Lehrpersonen unterschätzen also ihren „Sprechanteil“ in erheblichem Maß.

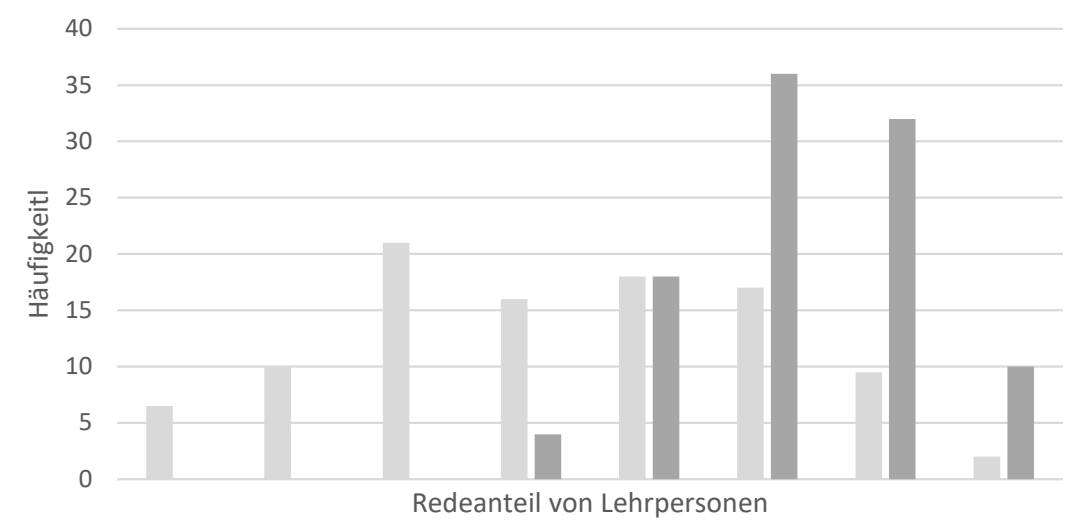

Selbsteinschätzung $\quad$ tatsächlich gemessen

Abb. 5 Redeanteil von Lehrpersonen im Unterricht (Helmke, 2017, S. 139) 
Diese „blinden Flecken“ können nur auf der Basis von Feedback aufgedeckt werden, da diese dadurch gekennzeichnet sind, dass sie nur durch externe Information sichtbar gemacht werden können. Den Abgleich der eigenen Perspektive mit der Perspektive von Feedbackgebenden sichtbar zu machen und die anschließende Nutzung der gewonnenen Information für die Entwicklung der Unterrichtsqualität ist ein wesentlicher Teil der Professionalisierung von Lehrpersonen - und dieser Teil erfordert von Lehrpersonen entsprechende Kompetenzen und Haltungen zum Umgang mit Feedback im eigenen Unterricht.

\section{Haltungen: \\ Voraussetzung für erfolgreiches Feedback}

So wirksam Feedback auch sein kann, es bleibt ein Möglichkeitsraum der Unterrichtsqualität. Damit es wirksam ist, erfordert es Kompetenz und Haltung vonseiten der Lehrperson: So reicht es nicht aus zu wissen (WISSEN), dass es Feedback auf verschiedenen Ebenen gibt, um auf diesen Ebenen ein lernwirksames Feedback geben zu können (KÖNNEN). Entscheidend ist vielmehr, dass Lehrpersonen ihre Rolle darin sehen, mit Lernenden ins Gespräch zu kommen und deren Rückmeldungen als wichtig erachten, um die eigene Professionalität weiterzuentwickeln. Es bedarf folglich einer entsprechenden Motivation (WOLLEN), die von dazugehörigen Motiven (WERTEN) getragen wird. Eine Lehrperson, die beispielsweise die Haltung hat, dass Lernende ihr zum Unterricht keine sinnvolle Rückmeldung geben können, wird mit diesen nicht ins Gespräch kommen, auch wenn sie wüsste, was zu tun wäre. Demgegenüber wird eine Lehrperson ihr Wissen und Können tagtäglich für diesen Dialog nutzen, wenn sie von der Haltung getragen wird, dass Lernende wichtige Ansprechpartner*innen für die Reflexion über die eigene Wirksamkeit sind. In folgendem Expertisemodell (K3W-Modell) im Anschluss an „Visible Learning“ (Abb. 6) wird dieser Gedanke zusammengefasst: 


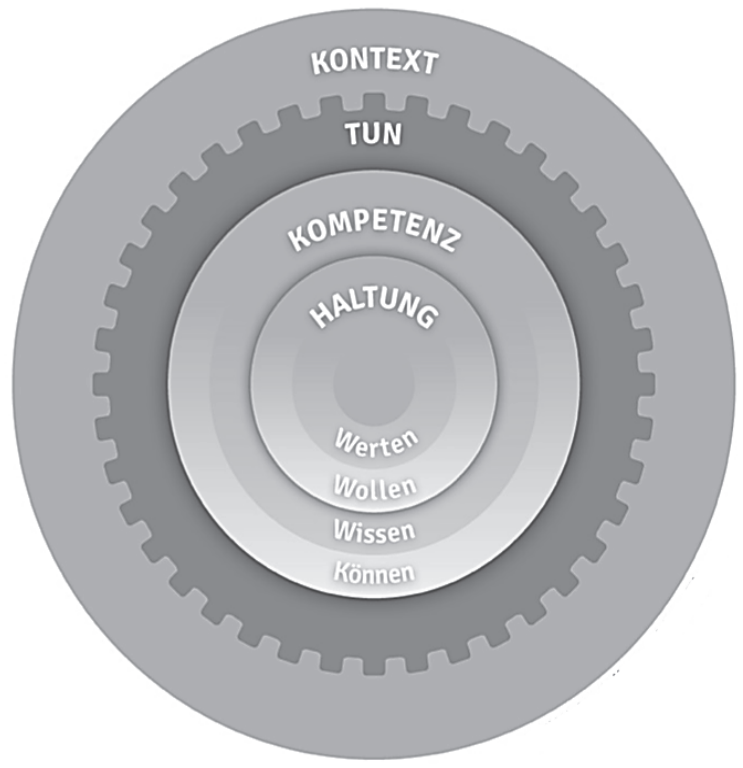

Abb. 6 Das K3W-Modell (Hattie \& Zierer, 2018b)

Ein weiterer Punkt für die Wirksamkeit von Feedback ist im Umgang mit dem erhaltenen Feedback zu sehen. Denn die Lehrperson muss das Feedback nicht nur annehmen, sondern es auch analysieren und damit umgehen können. Häufig wird sie dabei Feedback auf der Ebene der Aufgabe erhalten. Insofern zeigt sich ein professioneller Umgang mit Feedback von den Lehrpersonen darin, dass es gelingt, die erhaltenen Informationen auf der Ebene der Aufgabe zu nutzen, um daraus Rückschlüsse auf die Ebene des Prozesses und Konsequenzen für die Ebene der Selbstregulation ziehen zu können. Geben Schüler*innen beispielsweise die Rückmeldung, dass sie Übungsaufgaben nicht lösen konnten, so geben diese der Lehrperson Feedback auf der Ebene der Aufgabe. Eine Lehrperson ist nun gefordert, diese Erkenntnis zu nutzen und Strategien zu entwickeln, was im Unterricht ergänzt oder geändert werden muss, damit die Übungsaufgaben verstanden und gelöst werden können. Ein Gespräch mit den Lernenden über diese Situation ist häufig der nächste Schritt und liefert weitere Anhaltspunkte, was die Lehrperson ändern und anders machen kann. Spätestens an dieser Stelle wird sichtbar: Erfolgreiches Feedback bedeutet für Lehrpersonen auch immer, selbst Lernende zu sein und den eigenen Unterricht weiterzuentwickeln. 


\section{Handlungsmöglichkeiten für die Unterrichtspraxis}

Welche Folgerungen lassen sich nun für die Unterrichtspraxis ableiten? Wie bereits erwähnt, haben auch Lehrer*innen, wie jede andere Berufsgruppe, in Bezug auf ihre Berufsausübung sogenannte "blinde Flecken", wie sie im klassischen Modell des Johari-Fensters (s. Abb. 7 nach Luft \& Ingham, 1955) beschrieben werden. Dabei handelt es sich um Informationen, die zwar anderen Personen zugänglich sind, nicht aber den Akteuren, und die diesen ohne Rückmeldungen durch andere Personen auch nicht zugänglich gemacht werden können. Sie können von nahezu verschwindender bis hin zu essentieller Relevanz für den Unterrichtserfolg sein und vom häufigen Wiederholen eines bestimmten Füllwortes („Marotten“), über ungünstige nonverbale Signale bis zu der Tatsache, dass man Sachverhalte zu schnell oder zu unverständlich erklärt, reichen.

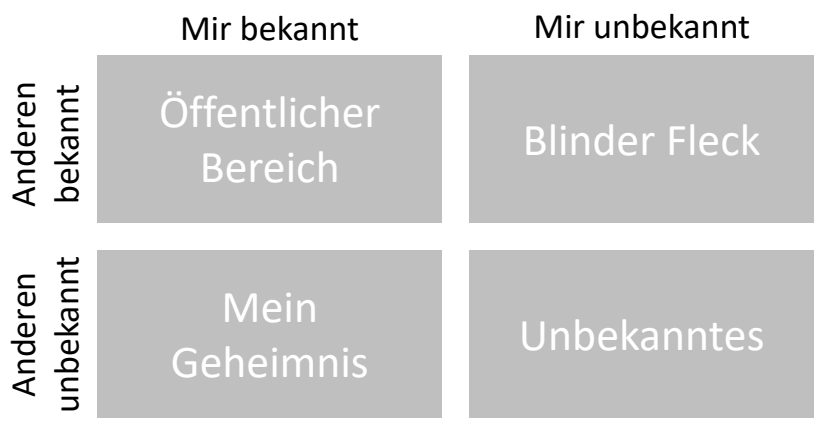

Abb. 7 Johari-Fenster

Diese blinden Flecken zu erkennen und durch Feedback sichtbar zu machen, ist ein wichtiger Beitrag für die Stärkung der Lehrperson und damit auch funktional für die Diagnostik und Verbesserung des eigenen Unterrichts. Hierzu bieten sich beispielsweise das Einholen von Feedback mit Hilfe von Fragebögen und die SWOT-Analyse an.

\section{Lernenden-Feedback einholen mit Hilfe von Fragebögen}

Die Steigerung der Unterrichtsqualität durch Feedback setzt bei der Umsetzung im Wesentlichen zwei Dinge voraus: Erstens werden Instrumente benötigt, mit denen auf möglichst objektive, reliabel und 
valide Art aussagekräftige Informationen eingeholt werden können (vgl. Wisniewski \& Zierer, 2017a) und zweitens bedarf es funktionaler Strategien, um die erhobenen Daten dann auch für eine tatsächliche Veränderung zu nutzen.

Eine zielführende Reflexion des eigenen Unterrichts setzt klare Kriterien der Lernwirksamkeit voraus. Diese liefern eine Orientierung für die Feedback-Gebenden, worauf zu achten ist und ermöglichen ein Gespräch über Unterricht, welches - abseits von vagen, normativen und persönlichkeitsbezogenen Aussagen - Verhalten beschreibt (Frommer \& Bovet, 1999). In der Schulpraxis erhalten Arbeitsgruppen, Steuergruppen oder ganze Kollegien von der Schulleitung gelegentlich den Auftrag, selbst Fragebögen zum Einholen von Feedback zum Unterricht zu entwickeln. Das Resultat sind dann sogenannte ad-hocInstrumente, die in erster Linie auf subjektiven Erfahrungen und persönlichen Einschätzungen Einzelner basieren. Im Gegensatz dazu bieten evidenzbasierte Fragebögen wie EMU (Evidenzbasierte Methoden der Unterrichtsdiagnostik und -entwicklung, Helmke et al., 2018) oder SEfU (Schüler als Experten für Unterricht, Universität Jena), Tripod's 7C (Ferguson \& Danielson, 2014) oder teaCh (Wisniewski \& Zierer, 2019) den Vorteil, dass die enthaltenen Items auf empirischen Forschungsergebnissen zur Lernwirksamkeit des Unterrichts basieren. Dass Lernende in der Lage sind, Merkmale der Unterrichtsqualität mithilfe solcher Instrumente verlässlich einzuschätzen, ist hinreichend belegt (Clausen, 2002; Kunter, 2005; Ferguson \& Danielson, 2014).

\section{SWOT-Analyse: Lernenden-Feedback umsetzen}

Das Einholen von Feedback per se ist keine Garantie für die Weiterentwicklung des Unterrichts. Wie bereits mehrfach betont wurde, kommt es darauf an, warum und wie Feedback eingeholt wird und wie damit umgegangen wird. Entscheidend ist der nächste Schritt, nämlich die Umsetzung der Ergebnisse des Feedbacks (Wisniewski \& Zierer, 2017b). Insbesondere ein kollegialer Austausch kann hierfür hilfreich sein (Roche \& Marsh, 2002; Penny \& Coe, 2004).

Eine Methode zum Umgang mit eingeholtem Feedback ist die SWOTAnalyse (Akronym für Strengths (Stärken), Weaknesses (Schwächen), Opportunities (Chancen) und Threats (Risiken), siehe Abb. 8. Sie hilft dabei, Rückmeldungen zu strukturieren, zielgerichtete Lösungen zu entwickeln und dabei schnelle und ungenaue Ratschläge (,hätte, könnte, sollte") zu vermeiden. 
SWOT-Analyse

\section{Gegenwart}

\begin{tabular}{l|l}
\hline Ressourcen (Strengths) & Schwierigkeiten (Weakness)
\end{tabular}

- Welche positiven Rückmeldungen gab - Welche(s) Problem(e) wurde(n) durch es von den Lernenden?

Womit sind die Lernenden zufrieden?

Was sollte ich beibehalten?

Worauf kann ich aufbauen?

Welche Rückmeldungen haben mich gefreut? die Lernenden identifiziert?

Was fehlt den Lernenden?

- Wo sind Veränderungen nötig?

- Was fällt mir noch schwer?

- Welche Rückmeldungen treffen mich?

Zukunft

\begin{tabular}{|l|l}
\hline Chancen (Opportunities) & Risiken (Threats)
\end{tabular}

- Welche Entwicklungsmöglichkeiten sehe ich?

- Wo sehe ich ungenutztes Potential?

- Was kann ich ausbauen?

- Was kann ich durch Investition erreichen?

- Welche Unterstützung kann ich nutzen?

- Welche Fehlentwicklungen befürchte ich?

- Wo stecken Risiken bei der Veränderung?

- Wo muss ich vorsichtig sein?

- Welche Probleme drohen, wenn ich nicht gegensteuere?

Abb. 8 SWOT-Analyse

\section{Schlussbetrachtung}

Welche Kriterien sind für erfolgreiches Feedback in Bildungsprozessen entscheidend? Zusammenfassend sind drei Punkte herauszustellen:

1. Erfolgreiches Feedback muss stets die Lernausgangslage der Schüler*innen in den Blick nehmen. Feedback an die Lernenden kann dann seine Wirkung entfalten, wenn es für jeden Lernenden individuell auf den drei Ebenen der Aufgabe, des Lernprozesses sowie der Selbstregulation gegeben wird.

2. Wichtiger als das Feedback, das Lehrpersonen geben, ist das Feedback, das Lehrpersonen erhalten. Dabei ist dreierlei zu beachten: (1) Zur Richtung: Feedback ist keine Einbahnstraße (von der Lehrperson an die Schüler*innen gerichtet), sondern auch in der Gegenrichtung (von den Schüler*innen an die Lehrperson) wirksam. (2) Zum Gegenstand: Feedback kann sich sowohl auf das Lehren (den Unterricht) als auch auf das eigene Lernen beziehen; beide Aspekte sind wichtig. Dies ergibt die in Abbildung 9 dargestellte Vierfeldertafel. Alle vier Quadranten haben dabei ihren je eigenen Wert. Ihre Gewichtung hängt im konkreten Fall vom Klassenkontext und der Lernsituation ab: 


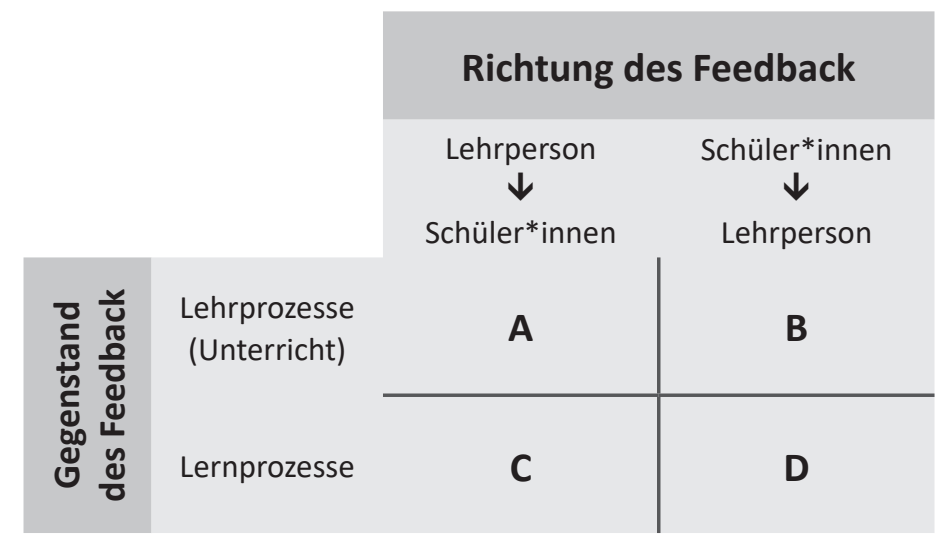

Abb. 9 Vierfeldertafel zu Feedback

(3) Zur Zeit: Schließlich gibt es auf der Zeitebene verschiedene Einsatzmöglichkeiten für kriterienorientiertes Feedback: Rückmeldungen während des laufenden Unterrichts, nach einer Unterrichtsstunde oder einer Lektion und kumulative Urteile über einen längeren Zeitraum hinweg (z. B. am Schuljahresende).

3. Die Kombination dieser drei Dimensionen (Richtung, Gegenstand, Zeit) eröffnet ein breites Spektrum von Szenarien für lernwirksames Feedback in der Praxis. Im Idealfall werden die individuellen Erfahrungen mit den Möglichkeiten - aber auch den Grenzen und Stolpersteinen - im Rahmen des Kollegiums oder in professionellen Lerngemeinschaften (Jahrgangsteam, Fachschaft, Qualitätszirkel) geteilt und gemeinsam besprochen.

Feedback in diesem Sinn wäre ein aussichtsreicher Schritt von der individuellen Professionalisierung in Richtung kooperativer Unterrichtsreflexion als eines Kernmerkmals einer Lernenden Schule und damit auch eines der vielversprechendsten Instrumente, um so etwas wie kollektive Wirksamkeitserwartungen als einen der einflussreichsten Faktoren überhaupt (vgl. Hattie \& Zierer, 2018a), realisieren zu können. Erfolgreiches Feedback ist mit Sicherheit nichts Leichtes, aber etwas sehr Wirksames. 


\section{Literatur}

Bastian, J., Combe, A. \& Langer, R. (2007). Feedback-Methoden: Erprobte Konzepte, evaluierte Erfahrungen. Weinheim: Beltz.

Buhren, C. G. \& Reitz, N. (2007). Evaluation an Schulen: Schülerselbstbeobachtung. In K.-O. Bauer (Hrsg.), Evaluation an Schulen (S. 53-80). Weinheim \& München: Juventa.

Clausen, M. (2002). Unterrichtsqualität: eine Frage der Perspektive? Empirische Analysen zur Übereinstimmungs-, Konstrukt- und Kriteriumsvalidität. Münster: Waxmann.

Ditton, H. \& Arnoldt, B. (2004). Schülerbefragungen zum Fachunterricht - Feedback an Lehrkräfte. Empirische Pädagogik, 18 (1), 115-139.

Ferguson, R. \& Danielson, C. (2014). How Framework for Teaching and Tripod 7Cs Evidence Distinguish Key Components of Effective Teaching. In T. Kane, K. Kerr \& R. Pianta (Eds.), Designing Teacher Evaluation Systems: New Guidance from the Measures of Effective Teaching Project (pp. 98-143). San Francisco: Jossey-Bass.

Frommer, H. \& Bovet, G. (1999). Praxis Lehrerberatung-Lehrerbeurteilung: Konzepte für Ausbildung und Schulaufsicht. Baltmannsweiler: Schneider.

Gärtner, H. (2013). Wirksamkeit von Schülerfeedback als Instrument der Selbstevaluation von Unterricht. In J. Hense, S. Rädiker, W. Böttcher \& T. Widmer (Hrsg.), Forschung über Evaluation. Bedingungen, Prozesse und Wirkungen (S. 107-124). Münster: Waxmann.

Hattie, J. (2018). Lernen sichtbar machen. Überarbeitete deutschsprachige Ausgabe von "Visible Learning" (4. Aufl.). Baltmannsweiler: Schneider.

Hattie, J. \& Zierer, K. (2018a). Visible Learning auf den Punkt gebracht. Baltmannsweiler: Schneider.

Hattie, J. \& Zierer, K. (2018b). Kenne deinen Einfluss! „Visible Learning“ für die Unterrichtspraxis (3. erw. Aufl.). Baltmannsweiler: Schneider.

Helmke, A. (2017). Unterrichtsqualität und Lehrerprofessionalität. Diagnose, Evaluation und Verbesserung des Unterrichts (7. Aufl., Schule weiterentwickeln - Unterricht verbessern. Orientierungsband). Seelze: Klett-Kallmeyer.

Helmke, A., Helmke, T., Lenske, G., Pham, G., Praetorius, A.-K., Schrader, F.-W. \& AdeThurow, M. (2018). EMU - Unterrichtsdiagnostik. Version 7.0 Kultusministerkonferenz. Landau: Universität Koblenz-Landau, Campus Landau. Verfügbar unter www.unterrichtsdiagnostik.info [25.05.2019].

Helmke, T., Helmke, A., Schrader, F.-W., Wagner, W., Nold, G. \& Schröder, K. (2008). Die Videostudie des Englischunterrichts. In DESI-Konsortium (Hrsg.), Unterricht und Kompetenzerwerb in Deutsch und Englisch. Ergebnisse der DESI-Studie (S. 345363). Weinheim: Beltz.

Kunter, M. (2005). Multiple Ziele im Mathematikunterricht. Münster: Waxmann.

Luft, J. \& Ingham, H. (1955). The Johari Window, a Graphic Model of Interpersonal Awareness. Proceedings of the Western Training Laboratory in Group Development, Los Angeles: UCLA.

Penny, A. R. \& Coe, R. (2004). Effectiveness of Consultation on Student Ratings Feedback: A meta-analysis. Review of Educational Research, 74 (2), 215-253.

Roche, L. A. \& Marsh, H. W. (2002). Teaching Self-Concept in Higher Education. In N. Hativa \& J. Goodyear (Eds.), Teacher Thinking, Beliefs and Knowledge in Higher Education (pp. 179-218). Dordrecht, Netherlands: Springer. 
SEfU - Schüler als Experten für Unterricht: SEfU in Kürze. Friedrich-Schiller-Universität Jena. Verfügbar unter https://www.sefu-online.de/index.php/ueber_sefu [25.05.2018].

Wisniewski, B. \& Zierer, K. (2017a). Schülerfeedback ist nicht gleich Schülerfeedback. PÄDAGOGIK, 11 (17), 38-42.

Wisniewski, B. \& Zierer, K. (2017b). Schülerfeedback - und dann? SchulVerwaltung Bayern - Fachzeitschrift für Schulentwicklung und Schulmanagement, 12, 324331.

Wisniewski, B. \& Zierer, K. (2019): Entwicklung eines Online-Fragebogens für Lernendenfeedback und erste Validierungsschritte. Erscheint in: Psychologie in Erziehung und Unterricht.

Zellner, J. (21.11.2013). Anstrengende Eltern, angestrengte Kinder. Optimierungswahn in der Schule. Frankfurter Allgemeine (FAZ). Verfügbar unter http://www. faz.net/aktuell/feuilleton/forschung-und-lehre/optimierungswahn-in-der-schuleanstrengende-eltern-angestrengte-kinder-12670511.html [12.11.2018].

Klaus Zierer, Dr. Prof., Ordinarius für Schulpädagogik an der Universität Augsburg. Arbeitsschwerpunkte: Visible Learning, Allgemeine Didaktik

klaus.zierer@phil.uni-augsburg.de

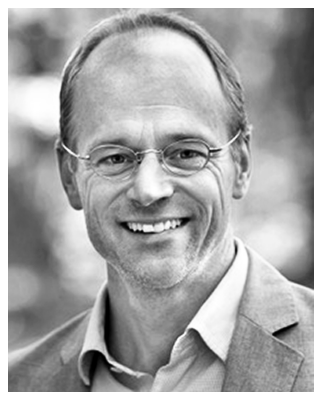

Benedikt Wisniewski, Dr., Staatlicher Schulpsychologe, wiss. Mitarbeiter am Lehrstuhl für Schulpädagogik an der Universität Augsburg. Arbeitsschwerpunkt: Feedback im Kontext Schule

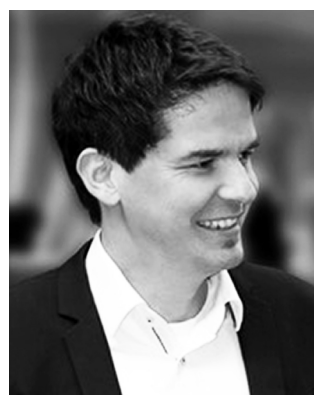


Christina Schatz, wiss. Mitarbeiterin am Lehrstuhl für Schulpädagogik der Universität Augsburg. Arbeitsschwerpunkte: Feedback, Lehrer*innenprofessionalität

christina.schatz@phil.uni-augsburg.de

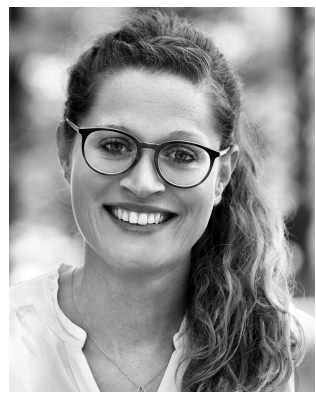

Denise Weckend, wiss. Mitarbeiterin am Lehrstuhl für Schulpädagogik der Universität Augsburg. Arbeitsschwerpunkte: Feedback, Lehrer*innenprofessionalität

denise.weckend@phil.uni-augsburg.de

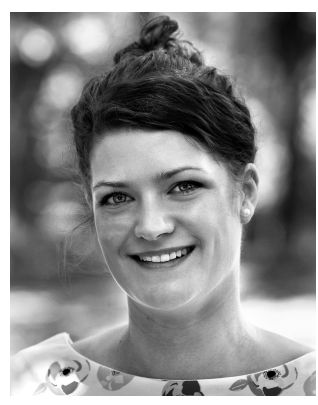

Andreas Helmke, Dr., Prof. für Entwicklungspsychologie und Bildungsforschung (em.), Universität Koblenz-Landau, Fachbereich Psychologie. Arbeitsschwerpunkte: Lehr-Lern-Forschung, Unterrichtsforschung, empirische Bildungsforschung

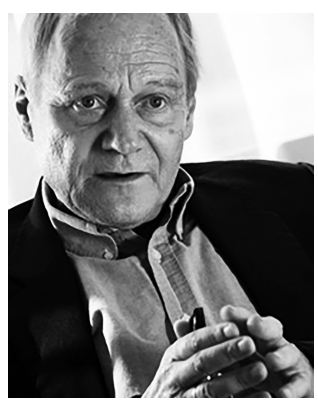

